Comment. Math. Helv. 72 (1997) 84-100

0010-2571/97/010084-17 \$1.50+0.20/0
(C) 1997 Birkhäuser Verlag, Basel

Commentarii Mathematici Helvetici

\title{
Symmetric and non-symmetric quantum Capelli polynomials
}

\author{
Friedrich Knop*
}

\begin{abstract}
We introduce families of symmetric and non-symmetric polynomials (the quantum Capelli polynomials) which depend on two parameters $q$ and $t$. They are defined in terms of vanishing conditions. In the differential limit $\left(q=t^{\alpha}\right.$ and $\left.t \rightarrow 1\right)$ they are related to Capelli identities. It is shown that the quantum Capelli polynomials form an eigenbasis for certain q-difference operators. As a corollary, we obtain that the top homogeneous part is a symmetric/non-symmetric Macdonald polynomial. Furthermore, we study the vanishing and integrality properties of the quantum Capelli polynomials.
\end{abstract}

Mathematics Subject Classification (1991). 05E05, 12H10, 39A70.

Keywords. Symmetric polynomials, Capelli identity, Macdonald polynomials, difference operators, Hecke algebras.

\section{Introduction}

Generalizing the classical Capelli identity has recently attracted a lot of interest $([\mathrm{HU}],[\mathrm{Ok}],[\mathrm{Ol}],[\mathrm{Sa}],[\mathrm{WUN}])$. In several of these papers it was realized, in various degrees of generality, that Capelli identities are connected with certain symmetric polynomials which are characterized by their vanishing at certain points. From this point of view, these polynomials have been constructed by Sahi [Sa] and were studied in $[\mathrm{KS}]$.

The purpose of this paper is twofold: we quantize the vanishing condition in a rather straightforward manner and obtain a family of symmetric polynomials which is indexed by partitions and which depends on two parameters $q, t$. As in $[\mathrm{KS}]$, their main feature is that they are non-homogeneous and one of our principal results states that the top degree terms are the Macdonald polynomials. It is an interesting problem whether these quantized Capelli polynomials are indeed connected with quantized Capelli identities (see [WUN]) as it is in the classical case.

* Partially supported by a grant of the NSF 
But the main progress over [KS] is the introduction of a family of non-symmetric polynomials which are also defined by vanishing conditions. They are nonhomogeneous and their top degree terms turns out to be the non-symmetric Macdonald polynomials. To prove this, we introduce certain difference operators of Cherednik type of which our polynomials are a simultaneous eigenbasis. Because of these operators, the non-symmetric functions are much easier to handle than the symmetric ones. Moreover, the latter can be obtained by a simple symmetrization process.

More specifically, the non-symmetric vanishing conditions are as follows: For $\lambda \in \Lambda:=\mathbb{N}^{n}$ let $|\lambda|:=\sum \lambda_{i}$ and let $w_{\lambda}$ be the shortest permutation such that $w_{\lambda}^{-1}(\lambda)$ is a partition (i.e., a non-increasing sequence). Let $q$ and $t$ be two formal parameters and consider the vector $\varrho:=\left(1, t^{-1}, t^{-2}, \ldots, t^{-n+1}\right)$. Then we prove that for every $\lambda \in \Lambda$ there is a polynomial $E_{\lambda}\left(z_{1}, \ldots, z_{n}\right)$, unique up to a scalar factor, which is of degree $|\lambda|$ and which satisfies the following condition:

$$
E_{\lambda}\left(q^{\mu} w_{\mu}(\varrho)\right)=0 \quad \text { for all } \mu \in \Lambda \text { with }|\mu| \leq|\lambda| \text { and } \mu \neq \lambda .
$$

We show that the affine Hecke algebra acts on these polynomials in a natural way and that there are Cherednik-type operators of which they are simultaneous eigenfunctions. This gives the link to the theory of homogeneous (symmetric or not) Macdonald polynomials.

Furthermore, in this paper we show two results which are in a way dual to each other: the polynomial $E_{\lambda}$ contains, in general, much fewer monomials than there are of degree $|\lambda|$ (triangularity) and it vanishes at many more points than required by definition (extra vanishing). The extra vanishing is expressed in terms of an order relation on $\Lambda$ which generalizes the order of partitions by inclusion of diagrams.

Later we prove that the quantized Capelli polynomials can be expressed in terms of their highest degree component (a Macdonald polynomial) and certain difference operators (inversion formula). This is used to transfer previous integrality results of mine, $[\mathrm{Kn}]$, to the case of Capelli polynomials.

In the final section, we discuss the transition from the quantum to the classical case. For this we put $t=q^{r}$ and let $q$ tend to one.

\section{Acknowledgements}

I would like to thank G. Heckman whose questions initiated the research to this paper and S. Sahi for many discussions on the case of the classical limit.

\section{The vanishing condition}

Let $\Lambda:=\mathbb{N}^{n}$ and $\Lambda^{+} \subseteq \Lambda$ the subset of partitions. For $\lambda=\left(\lambda_{i}\right) \in \Lambda$ we write $|\lambda|:=\sum_{i} \lambda_{i}$ and $l(\lambda):=\max \left\{i \mid \lambda_{i} \neq 0\right\}($ with $l(0)=0)$.

Let $k$ be a field of characteristic zero and $\mathcal{P}:=k\left[z_{1}, \ldots, z_{n}\right]$ the polynomial ring and $\mathcal{P}^{\prime}:=k\left[z_{1}, z_{1}^{-1}, \ldots, z_{n}, z_{n}^{-1}\right]$ the ring of Laurent polynomials. To each 
$\lambda \in \Lambda$ corresponds a monomial $z^{\lambda}=\prod_{i} z_{i}^{\lambda_{i}}$. Fix two non-zero elements $q$ and $t$ of $k$. Throughout the paper we assume that $q^{a} t^{b} \neq 1$ for all integers $a, b \geq 1$.

The symmetric group $W:=S_{n}$ acts in the obvious way on $\Lambda$ and $\mathcal{P}$. Every $\lambda \in \Lambda$ contains a unique partition $\lambda^{+}$in its $W$-orbit. Let $w_{\lambda} \in W$ be the shortest permutation such that $w_{\lambda}\left(\lambda^{+}\right)=\lambda$. For all $\lambda \in \Lambda$ and $x=\left(x_{i}\right), y=\left(y_{i}\right) \in k^{n}$ let $q^{\lambda}:=\left(q^{\lambda_{i}}\right)$ and $x y:=\left(x_{i} y_{i}\right)$. Consider the element $\varrho:=\left(1, t^{-1}, t^{-2}, \ldots, t^{-n+1}\right) \in$ $k^{n}$.

For every $\lambda \in \Lambda$ we define $\bar{\lambda}:=w_{\lambda}\left(q^{\lambda^{+}} \varrho\right)$. More concretely, $\bar{\lambda}_{i}=q^{\lambda_{i}} t^{-k_{i}}$ where

$$
k_{i}=k_{i}(\lambda)=\#\left\{j=1, \ldots, i-1 \mid \lambda_{j} \geq \lambda_{i}\right\}+\#\left\{j=i+1, \ldots, n \mid \lambda_{j}>\lambda_{i}\right\} .
$$

The following simple lemma is fundamental:

2.1. Lemma. For $\lambda \in \Lambda$ with $\lambda_{n} \neq 0$ let $\lambda^{*}:=\left(\lambda_{n}-1, \lambda_{1}, \ldots, \lambda_{n-1}\right)$. Then $\overline{\lambda^{*}}=\left(\bar{\lambda}_{n} / q, \bar{\lambda}_{1}, \ldots, \bar{\lambda}_{n-1}\right)$.

Proof. Follows easily from the definition.

2.2. Theorem. For $d \in \mathbb{N}$ let $S(n, d)$ be the set of all $\bar{\lambda}$ where $\lambda \in \Lambda$ and $|\lambda| \leq d$. Let $\bar{f}: S(n, d) \rightarrow k$ be a mapping. Then there exists a unique polynomial $f \in \mathcal{P}$ of degree at most $d$ such that $f(z)=\bar{f}(z)$ for all $z \in S(n, d)$.

Proof. The cardinality of $S(n, d)$ equals the dimension of the space of polynomials of degree at most $d$. Hence existence of $f$ will imply its uniqueness.

To show existence, we proceed by induction on $n+d$. Every polynomial can be uniquely written as

$$
f\left(z_{1}, \ldots, z_{n}\right)=g\left(z_{1}, \ldots, z_{n-1}\right)+\left(z_{n}-t^{-n+1}\right) h\left(z_{n} / q, z_{1}, z_{2}, \ldots, z_{n-1}\right) .
$$

Consider first the set $S_{0}$ of $\bar{\lambda} \in S(n, d)$ with $\lambda_{n}=0$, i.e., $\bar{\lambda}_{n}=t^{-n+1}$. Then, as $z$ runs through $S_{0}, z^{\prime}:=\left(z_{1}, \ldots, z_{n-1}\right)$ will run through $S(n-1, d)$. By induction, one can choose $g$ such that $f$ takes the required values at $S_{0}$.

Consider now the set $S_{1}$ of remaining points $\bar{\lambda}$ with $\lambda_{n} \neq 0$. By the lemma, as $z$ runs through $S_{1},\left(z_{n} / q, z_{1}, z_{2}, \ldots, z_{n-1}\right)$ will run through $S(n, d-1)$. The factor $z_{n}-t^{-n+1}=\bar{\lambda}_{n}-t^{-n+1}$ is not zero by the choice of $q$ and $t$. By induction, we can find $h$ of degree at most $d-1$ with arbitrary values at $S(n, d-1)$. So $f$ exists.

There is also a statement for symmetric polynomials which is the quantized version of a theorem of Sahi [Sa]. For $\lambda \in \Lambda^{+}$let $m_{\lambda}$ be the corresponding monomial symmetric polynomial.

2.3. Theorem. For $d \in \mathbb{N}$ let $S^{+}(n, d)$ be the set of all $\bar{\lambda}=q^{\lambda} \varrho$ where $\lambda \in \Lambda^{+}$ and $|\lambda| \leq d$. Let $\bar{f}: S^{+}(n, d) \rightarrow k$ be a mapping. Then there exists a unique polynomial $f \in \mathcal{P}$ of degree at most $d$ such that $f(z)=\bar{f}(z)$ for all $z \in S^{+}(n, d)$. 
Proof. The proof is completely analogous to that in [Sa]. Again, only existence has to be proved. Let $g \mapsto g^{+}$be the linear map from symmetric polynomials in $n-1$ variables to those with $n$ variables which sends $m_{\lambda}\left(z_{1}-t^{-n+1}, \ldots, z_{n-1}-t^{-n+1}\right)$ to $m_{\lambda, 0}\left(z_{1}-t^{-n+1}, \ldots, z_{n}-t^{-n+1}\right)$. This map preserves degrees and satisfies $g^{+}\left(z_{1}, \ldots, z_{n-1}, t^{-n+1}\right)=g\left(z_{1}, \ldots, z_{n-1}\right)$. We construct $f$ by setting

$$
f\left(z_{1}, \ldots, z_{n}\right)=g^{+}\left(z_{1}, \ldots, z_{n}\right)+\prod_{i=1}^{n}\left(z_{i}-t^{-n+1}\right) h\left(z_{1} / q, \ldots, z_{n} / q\right),
$$

where $g^{+}$and $h$ are symmetric. Then, as above, evaluation at $\bar{\lambda} \in S^{+}(n, d)$ with $\lambda_{n}=0$ gives $g$. Evaluation at the other points gives $h$.

We obtain the following Theorem/Definition:

2.4. Theorem. a) For every $\lambda \in \Lambda$ there is a unique polynomial $E_{\lambda}$ with $E_{\lambda}(\bar{\mu})=$ 0 for all $\mu \in \Lambda$ with $|\mu| \leq|\lambda|, \mu \neq \lambda$ and which has an expansion $E_{\lambda}=\sum_{\mu} e_{\lambda \mu} z^{\mu}$ with $e_{\lambda \lambda}=1$.

b) For every $\lambda \in \Lambda^{+}$there is a unique symmetric polynomial $P_{\lambda}$ with $P_{\lambda}(\bar{\mu})=0$ for all $\mu \in \Lambda^{+}$with $|\mu| \leq|\lambda|, \mu \neq \lambda$ and which has an expansion $P_{\lambda}=\sum_{\mu} p_{\lambda \mu} m_{\mu}$ with $p_{\lambda \lambda}=1$.

Proof. By Theorem 2.2, there is a polynomial $E_{\lambda}$ satisfying the vanishing condition with $E_{\lambda}(\bar{\lambda}) \neq 0$. We have to show that it contains $z^{\lambda}$ with a non-zero coefficient. Let

$$
E_{\lambda}\left(z_{1}, \ldots, z_{n}\right)=g\left(z_{1}, \ldots, z_{n-1}\right)+\left(z_{n}-t^{-n+1}\right) h\left(z_{n} / q, z_{1}, z_{2}, \ldots, z_{n-1}\right) .
$$

If $\lambda_{n}=0$, then $g=E_{\lambda^{\prime}}$ with $\lambda^{\prime}=\left(\lambda_{1}, \ldots, \lambda_{n-1}\right)$. By induction, $g$, and therefore $f$, contains $z^{\lambda}$. If $\lambda_{n} \neq 0$ then $g=0$ and $h$ a multiple of $E_{\lambda^{*}}$. We conclude again by induction.

The proof in the symmetric case is analogous.

Our proof actually gives a bit more. Consider the operators

$$
\Delta f\left(z_{1}, \ldots, z_{n}\right):=f\left(z_{n} / q, z_{1}, \ldots, z_{n-1}\right),
$$

and $\Phi:=\left(z_{n}-t^{-n+1}\right) \Delta$. Then we have

2.5. Corollary. For $\lambda \in \Lambda$ with $\lambda_{n} \neq 0$ let $\lambda^{*}=\left(\lambda_{n}-1, \lambda_{1}, \ldots, \lambda_{n-1}\right)$. Then $E_{\lambda}=q^{\lambda_{n}-1} \Phi\left(E_{\lambda^{*}}\right)$

For $\lambda \in \Lambda$ let $\mathcal{P}_{\lambda} \subseteq \mathcal{P}$ be the set of polynomials of degree at most $|\lambda|$ which vanish at all $\bar{\mu}$ with $|\mu| \leq|\lambda|$ and $\mu \notin W \lambda$. Of course, $\mathcal{P}_{\lambda}$ depends only on $\Lambda^{+}$.

2.6. Corollary. We have $\mathcal{P}=\oplus_{\lambda \in \Lambda^{+}} \mathcal{P}_{\lambda}$. The set $\left\{E_{w \lambda} \mid w \in W\right\}$ forms a basis of $\mathcal{P}_{\lambda}$. Moreover, $\mathcal{P}_{\lambda} \cap \mathcal{P}^{W}=k P_{\lambda}$. 
Proof. Follows directly from the definition.

We conclude this section by giving two examples. For $k \in \mathbb{N}$ we define the $q$ factorial polynomial as $[z ; k]_{q}:=(z-1)(z-q) \ldots\left(z-q^{k-1}\right)$. Then the following is obvious.

2.7. Proposition. Let $t=1$. Then $E_{\lambda}(z ; q, 1)=\left[z_{1} ; \lambda_{1}\right]_{q} \ldots\left[z_{n} ; \lambda_{n}\right]_{q}$ and $P_{\lambda}(z ; q, 1)$ is the symmetrization of it.

Now we consider the case $t=q$ in the symmetric case. For $\lambda \in \Lambda^{+}$we define the $q$-factorial Schur function as $\mathfrak{s}_{\lambda}(z ; q):=a^{-1} \operatorname{det}\left[z_{i} ; \lambda_{j}+n-j\right]_{q}$ where $a=\prod_{i<j}\left(z_{i}-z_{j}\right)$ is the Vandermonde determinant.

2.8. Proposition. Let $t=q$. Then $P_{\lambda}(z ; q, q)=q^{-(n-1)|\lambda|} \mathfrak{s}_{\lambda}\left(q^{n-1} z ; q\right)$.

Proof. The proof is the same as in the classical case [KS] Prop.3.3.

\section{Hecke operators}

In this section, we are constructing operators which are adapted to the decomposition $\mathcal{P}=\oplus_{\lambda \in \Lambda^{+}} \mathcal{P}_{\lambda}$. Let $s_{i} \in W$ be the $i$-th simple reflection. Then

$$
N_{i}:=\left(z_{i}-z_{i+1}\right)^{-1}\left(1-s_{i}\right)
$$

is a well defined operator on $\mathcal{P}$. We define the Hecke operators

$$
\begin{aligned}
& H_{i}:=s_{i}-(1-t) N_{i} z_{i}=t s_{i}-(1-t) z_{i} N_{i}, \\
& \bar{H}_{i}:=s_{i}-(1-t) z_{i+1} N_{i}=t s_{i}-(1-t) N_{i} z_{i+1} .
\end{aligned}
$$

They satisfy the relations $H_{i}-\bar{H}_{i}=t-1$ and $H_{i} \bar{H}_{i}=t$. In particular, both $H_{i}$ and $-\bar{H}_{i}$ satisfy the equation $(x+1)(x-t)=0$. In addition the braid relations hold

$$
\begin{array}{rlrl}
H_{i} H_{i+1} H_{i} & =H_{i+1} H_{i} H_{i+1} & i=1, \ldots, n-2 \\
H_{i} H_{j} & =H_{j} H_{i} & |i-j|>1
\end{array}
$$

This means that the algebra $\mathcal{H}$ generated by the $H_{i}$ is the Hecke algebra of the root system $A_{n-1}$. For details see [Ch], [M2], or [Kn].

3.1. Lemma. Let $\mu \in \Lambda$ and $f \in \mathcal{P}^{\prime}$. Then $H_{i} f(\bar{\mu})$ and $\bar{H}_{i} f(\bar{\mu})$ are linear combinations of $f(\bar{\mu})$ and $f\left(\overline{s_{i} \mu}\right)$ where the coefficients are independent of $f$.

Proof. We have

$$
\bar{H}_{i} f(\bar{\mu})=\left[s_{i}-(1-t) z_{i+1} N_{i}\right] f(\bar{\mu})=\frac{(t-1) \bar{\mu}_{i+1}}{\bar{\mu}_{i}-\bar{\mu}_{i+1}} f(\bar{\mu})+\frac{\bar{\mu}_{i}-t \bar{\mu}_{i+1}}{\bar{\mu}_{i}-\bar{\mu}_{i+1}} f\left(s_{i} \bar{\mu}\right) .
$$


If $\mu_{i} \neq \mu_{i+1}$ then $s_{i} \bar{\mu}=\overline{s_{i} \mu}$. Otherwise, $\bar{\mu}_{i}-t \bar{\mu}_{i+1}=0$ by definition of $\bar{\mu}$.

3.2. Corollary. For every $\lambda \in \Lambda^{+}$holds $\mathcal{H} \mathcal{P}_{\lambda} \subseteq \mathcal{P}_{\lambda}$.

3.3. Corollary. Let $X$ be an operator in the algebra $\mathcal{A}$ generated by the $H_{i}, z_{i}$, and $\Phi$ (respectively in the both sided ideal $\mathcal{A} \Phi \mathcal{A}$ ). Let $\lambda \in \Lambda$ and $f \in \mathcal{P}^{\prime}$. Then $X f(\bar{\lambda})$ is a linear combination of $f(\bar{\mu})$ where $\mu \in \Lambda$ and $|\mu| \leq|\lambda|$ (respectively $|\mu|<|\lambda|)$.

3.4. Corollary. Let $\lambda \in \Lambda$ with $\lambda_{i}=\lambda_{i+1}$. Then $\bar{H}_{i}\left(E_{\lambda}\right)=E_{\lambda}$ and $H_{i}\left(E_{\lambda}\right)=$ $t E_{\lambda}$.

Proof. Let us consider $E:=H_{i}\left(E_{\lambda}\right)$ and $\mu \in \Lambda$ with $|\mu| \leq|\lambda|$ and $\mu \neq \lambda$. Since also $s_{i} \mu \neq \lambda$, we obtain $E_{\lambda}(\bar{\mu})=E_{\lambda}\left(\overline{s_{i} \mu}\right)=0$, hence $E(\bar{\mu})=0$. This means that $E$ is a multiple of $E_{\lambda}$. Evaluation at $z=\bar{\lambda}$ implies that the factor is $t$.

For $i=1, \ldots, n$ we define the Cherednik operators

$$
\xi_{i}^{-1}=\bar{H}_{i} \ldots \bar{H}_{n-1} \Delta H_{1} \ldots H_{i-1} .
$$

Their relevance will become clear later. Furthermore, we define the operators

$$
\Xi_{i}:=z_{i}^{-1}+z_{i}^{-1} H_{i} \ldots H_{n-1} \Phi H_{1} \ldots H_{i-1}
$$

which are a priori only well-defined on Laurent polynomials. The following relations are easily established (see $[\mathrm{Kn}] \S 3$ )

$$
H_{i} \Xi_{i}=\Xi_{i+1} \bar{H}_{i}, \quad i=1, \ldots, n-1 ; \quad H_{i} \Xi_{j}=\Xi_{j} H_{i}, \quad j \neq i, i+1 .
$$

3.5. Lemma. The operators $\Xi_{i}$ act on $\mathcal{P}$.

Proof. Since $\Xi_{i}=t^{-1} \bar{H}_{i} \Xi_{i+1} \bar{H}_{i}$ it suffices to consider the case $i=n$. Because

$$
\Xi_{n}:=\left(z_{n}-t^{-n+1}\right) z_{n}^{-1}\left(\xi_{n}^{-1}-t^{n-1}\right)+t^{n-1},
$$

the assertion follows from the following claim:

$$
\xi_{n}^{-1} f=t^{n-1} f \bmod z_{n} \quad \text { for all } f \in \mathcal{P}
$$

We write $f \equiv g$ for $f=g \bmod z_{n}$. First we show by induction

$$
\text { (*) } \quad \Delta H_{1} \ldots H_{i} f \equiv t^{i-1} \Delta s_{1} \ldots s_{i} f .
$$

We have $\Delta H_{1} \ldots H_{i} f \equiv t^{i-2} \Delta s_{1} \ldots s_{i-1}\left(t s_{i}-(1-t) z_{i} N_{i}\right) f$. Then $(*)$ follows from $\Delta s_{1} \ldots s_{i-1} z_{i}=q^{-1} z_{n} \Delta s_{1} \ldots s_{i-1}$. 
For $i=n-1,(*)$ reads $\xi_{n}^{-1} f \equiv t^{n-1} \Delta s_{1} \ldots s_{n-1} f$. The claim follows from

$$
\Delta s_{1} \ldots s_{n-1} f(z)=f\left(z_{1}, \ldots, z_{n-1}, z_{n} / q\right) \equiv f(z) .
$$

The $\Xi_{i}$ are inhomogeneous versions of the Cherednik operators. Observe, that $\operatorname{deg} \Xi_{i}(f) \leq \operatorname{deg} f$ for all $f \in \mathcal{P}$. The main result of this paper is

3.6. Theorem. The $E_{\lambda}$ form a simultaneous eigenbasis for the $\Xi_{i}$. More precisely, $\Xi_{i}\left(E_{\lambda}\right)=\bar{\lambda}_{i}^{-1} E_{\lambda}$ for all $\lambda \in \Lambda$ and $i=1, \ldots, n$.

Proof. Let $d:=|\lambda|$ and write $\Xi_{i}=z_{i}^{-1}+X_{i}$. Then $\Xi_{i}\left(E_{\lambda}\right)=z_{i}^{-1} E_{\lambda}+X_{i}\left(E_{\lambda}\right)$. Since $E_{\lambda}$ vanishes in $S(n, d-1)$, Lemma 3.1 implies that $X_{i}\left(E_{\lambda}\right)$ vanishes in $S(n, d)$. Hence, $\Xi_{i}\left(E_{\lambda}\right)$ vanishes for all $\mu \in S(n, d)$ with $\mu \neq \lambda$. This implies that $\Xi_{i}\left(E_{\lambda}\right)=c E_{\lambda}$ for some $c \in k$. Evaluation at $z=\bar{\lambda}$ implies $c=\bar{\lambda}_{i}^{-1}$.

3.7. Corollary. The operators $\Xi_{1}, \ldots, \Xi_{n}$ commute pairwise.

Next we consider the non-homogeneous analogues of the Macdonald operators:

3.8. Corollary. Let $p \in \mathcal{P}^{W}$. Then $\Xi_{p}:=p\left(\Xi_{1}, \ldots, \Xi_{n}\right)$ commutes with all $H_{i}$. Moreover, $\Xi_{p}\left(P_{\lambda}\right)=p\left(\bar{\lambda}^{-1}\right) P_{\lambda}$.

Proof. $\Xi_{p}$ acts on $\mathcal{P}_{\lambda}$ as scalar multiplication by $p\left(\bar{\lambda}^{-1}\right)$.

3.9. Theorem. Let $\bar{E}_{\lambda}$ be the top homogeneous part of $E_{\lambda}$. Then $\xi_{i}^{-1}\left(\bar{E}_{\lambda}\right)=$ $\bar{\lambda}_{i}^{-1} \bar{E}_{\lambda}$. This means, that $\bar{E}_{\lambda}$ is a non-symmetric Macdonald polynomial. Similarly, the top homogeneous part $\bar{P}_{\lambda}$ of $P_{\lambda}$ is a symmetric Macdonald polynomial.

Proof. We have $\Xi_{n}=\xi_{n}^{-1}$ plus terms which decrease the degree. Therefore, also $\Xi_{i}=\xi_{i}^{-1}$ plus degree decreasing operators. The theorem follows since the Macdonald polynomials are characterized as eigenfunctions of the $\xi_{i}$.

There is a (partial) order relation on $\Lambda$. First, recall the usual order on the set $\Lambda^{+}$: we say $\lambda \geq \mu$ if

$$
\lambda_{1}+\lambda_{2}+\ldots+\lambda_{i} \geq \mu_{1}+\mu_{2}+\ldots+\mu_{i} \quad \text { for all } i=1, \ldots, n
$$

This order relation is extended to all of $\Lambda$ as follows. For every $\lambda \in \Lambda$ there is a unique partition $\lambda^{+}$in the orbit $W \lambda$. For all permutations $w \in W$ with $\lambda=w \lambda^{+}$ there is a unique one, denoted by $w_{\lambda}$, of minimal length. We define $\lambda \geq \mu$ if either 
$\lambda^{+}>\mu^{+}$or $\lambda^{+}=\mu^{+}$and $w_{\lambda} \leq w_{\mu}$ in the Bruhat order of $W$. In particular, $\lambda^{+}$ is the unique maximum of $W \lambda$.

3.10. Lemma. The operators $\Xi_{i}$ are triangular. More precisely, $\Xi_{i}\left(z^{\lambda}\right)=\bar{\lambda}_{i}^{-1} z^{\lambda}+$ $\sum_{\mu<\lambda} c_{\lambda \mu} z^{\mu}$.

Proof. For this we write $\Xi_{i}=z_{i}^{-1}+\bar{H}_{i} \ldots \bar{H}_{n-1}\left(1-t^{-n+1} z_{n}^{-1}\right) \Delta H_{1} \ldots H_{i-1}=$ $\xi_{i}^{-1}+Y_{i}$ where $Y_{i}=z_{i}^{-1}-t^{-n+1} \bar{H}_{i} \ldots \bar{H}_{n-1} z_{n}^{-1} \Delta H_{1} \ldots H_{i-1}$. It is well known that $\xi_{i}^{-1}$ is triangular (see [M2]) with the given coefficient of $z^{\lambda}$. Since $Y_{i}$ decreases the degree it suffices to show that $\mu^{+}<\lambda^{+}$for each monomial $z^{\mu}$ occuring in $Y_{i}\left(z^{\lambda}\right)$. But that is easy to check.

3.11. Theorem. For every $\lambda \in \Lambda$ there is the expansion $E_{\lambda}=z^{\lambda}+\sum_{\substack{\mu \in \Lambda \\ \mu<\lambda}} e_{\lambda \mu} z^{\mu}$. Similarly, if $\lambda \in \Lambda^{+}$then $P_{\lambda}=m_{\lambda}+\sum_{\substack{\mu \in \Lambda^{+} \\ \mu<\lambda}} p_{\lambda \mu} m_{\mu}$.

Proof. By the triangularity and diagonalizability of $\Xi_{i}$ there must be an eigenfunction of the stated form with eigenvalue $\bar{\lambda}_{i}^{-1}$. Thus, it equals $E_{\lambda}$.

\section{The extra vanishing theorem}

We are going to introduce another (partial) order relation on $\Lambda$ which extends the containment relation on partitions. Let $\lambda, \mu \in \Lambda$. Then we say $\lambda \preccurlyeq \mu$ if there is a permutation $\pi \in W$ such that $\lambda_{i}<\mu_{\pi(i)}$ if $i<\pi(i)$ and $\lambda_{i} \leq \mu_{\pi(i)}$ if $i \geq \pi(i)$. In this case we call $\pi$ a defining permutation for $\lambda \preccurlyeq \mu$.

4.1. Lemma. If $\lambda \preccurlyeq \mu$ and $|\lambda| \geq|\mu|$ then $\lambda=\mu$.

Proof. All inequalities $\lambda_{i} \leq \mu_{\pi(i)}$ must the equalities. This can only happen if $i \geq \pi(i)$ for all $i$ which implies $\pi=$ id and $\lambda=\mu$.

If $\lambda$ and $\mu$ are partitions then $\lambda \preccurlyeq \mu$ is just the usual inclusion relation among diagrams but in general " $\preccurlyeq$ " it is finer than " $\subseteq$ ".

We proceed by describing the minimal elements lying above $\lambda$. For a subset $I=\left\{i_{1}, \ldots, i_{r}\right\}$ of $\{1, \ldots, n\}$ with $i_{1}<\ldots<i_{r}$ we define the cyclic permutation $\pi_{I}: i_{r} \mapsto i_{r-1} \mapsto \ldots \mapsto i_{1} \mapsto i_{r}$ and $c_{I}(\lambda):=\mu \in \Lambda$ by

$$
\begin{aligned}
& \mu_{\pi_{I}(i)}=\lambda_{i} \quad i \in I, \quad i \neq i_{1} ; \\
& \mu_{i_{r}}=\lambda_{i_{1}}+1 ; \\
& \mu_{i}=\lambda_{i} \quad i \notin I .
\end{aligned}
$$

Clearly, $\lambda \prec c_{I}(\lambda)$ with the defining permutation $\pi_{I}$. Conversely, 
4.2. Lemma. Let $\lambda, \mu \in \Lambda$ with $\lambda \prec \mu$. Then there is $I$ such that $c_{I}(\lambda) \preccurlyeq \mu$.

Proof. Let $\pi$ be a defining permutation of $\lambda \preccurlyeq \mu$. If $\pi=$ id then $\lambda_{i}<\mu_{i}$ for some $i$ and we can choose $I=\{i\}$. Assume from now on $\pi \neq$ id.

Let $i_{1}$ be minimal with $\pi\left(i_{1}\right) \neq i_{1}$. Then necessarily $\pi\left(i_{1}\right)>i_{1}$. Put $i_{k}:=$ $\pi^{k-1}\left(i_{1}\right)$ and let $r \geq 2$ be minimal with $i_{r+1}>i_{r}$. Then we have $i_{1} \leq i_{r}<\ldots<i_{2}$. Now we take $I:=\left\{i_{1}, i_{r}, \ldots, i_{2}\right\}$ (note that $i_{r}$ might be equal to $i_{1}$ ). Then, it follows from $\mu_{i_{2}} \geq \lambda_{i_{1}}+1, \mu_{i_{3}} \geq \lambda_{i_{2}}$ etc. that $c_{I}(\lambda) \preccurlyeq \mu$ with defining permutation $\pi \pi_{I}^{-1}$.

The next lemma shows that one doesn't have to check all permutations to show $\lambda \preccurlyeq \mu$.

4.3. Lemma. Let $\lambda, \mu \in \Lambda$ with $\lambda \preccurlyeq \mu$. Then $\pi=w_{\mu} w_{\lambda}^{-1}$ is a defining permutation.

Proof. First note that $\pi$ is the permutation with $k_{i}(\lambda)=k_{\pi(i)}(\mu)$ for all $i$ (with $k_{i}$ as in section 2). Fixing $\pi$ like that certainly defines a new order relation $\preccurlyeq^{\prime}$ which is coarser than $\preccurlyeq$. To show that these relations coincide it suffices to show $\lambda \preccurlyeq{ }^{\prime} c_{I}(\lambda)$ for all $I$.

For this we may assume that $I$ is maximal among all $J$ with $\mu:=c_{I}(\lambda)=c_{J}(\lambda)$. This means that

$$
\begin{aligned}
& \lambda_{i} \neq \lambda_{i_{1}} \quad \text { for } i=1, \ldots, i_{1}-1 ; \\
& \begin{array}{l}
\lambda_{i} \neq \lambda_{i_{2}} \\
\text { etc. }
\end{array} \quad \text { for } i=i_{1}+1, \ldots, i_{2}-1 ; \\
& \lambda_{i} \neq \lambda_{i_{r}} \quad \text { for } i=i_{r-1}+1, \ldots, i_{r}-1 ; \\
& \lambda_{i} \neq \lambda_{i_{1}}+1 \text { for } i=i_{r}+1, \ldots, n .
\end{aligned}
$$

In this case one verifies easily

$$
k_{i_{j}}(\mu)=k_{i_{j+1}}(\lambda) \quad j=1, \ldots, r-1 ; \quad k_{i_{r}}(\mu)=k_{i_{1}}(\lambda)
$$

and $k_{i}(\mu)=k_{i}(\lambda)$ otherwise, i.e., $w_{\mu} w_{\lambda}^{-1}=\pi_{I}$.

Definition. A subset $S \subseteq \Lambda$ is called closed if $\lambda \in S$ and $\lambda \preccurlyeq \mu$ implies $\mu \in S$. If that is the case let $I_{S} \subseteq \mathcal{P}$ be the ideal of functions $f$ which vanish in all $\bar{\lambda}$ with $\lambda \in \Lambda \backslash S$.

4.4. Theorem. Let $S \subseteq \Lambda$ be a closed subset. Then $\Xi_{i}\left(I_{S}\right) \subseteq I_{S}$ for all $i=$ $1, \ldots, n$.

Proof. Let $\mu \in \Lambda \backslash S$ and $f \in I_{S}$. We have to show that $\Xi_{i} f(\bar{\mu})=0$. The definition of $\Xi_{i}$ shows that $\Xi_{i} f(\bar{\mu})$ is a linear combination of $f(\bar{\mu})$ and terms of the form

$$
y:=\sigma_{i} \ldots \sigma_{n-1} \Delta \sigma_{1} \ldots \sigma_{i-1} f(\bar{\mu})
$$


where each operator $\sigma_{j}$ is either $s_{i}$ or 1 . This shows that $y=f(\bar{\lambda})$ with $\mu=c_{I}(\lambda)$ for some $I$ and $\lambda \in \Lambda$. Since $S$ is closed we have $\lambda \in \Lambda \backslash S$ and therefore, $y=0$.

Now we show the extra vanishing theorem:

4.5. Theorem. Let $\lambda, \mu \in \Lambda$ with $\lambda \npreceq \mu$. Then $E_{\lambda}(\bar{\mu})=0$.

Proof. Consider the closed subset $S=\{\nu \in \Lambda \mid \lambda \preccurlyeq \nu\}$. We have to show $E_{\lambda} \in I_{S}$. For generic $q$ and $t$ there is a function $f \in I_{S}$ with $f(\bar{\lambda}) \neq 0$. Indeed, take for example

$$
f(z):=\prod_{\pi \in W}\left[\prod_{i<\pi(i)} \varphi_{\lambda_{i}+1}\left(\bar{\lambda}_{i}^{-1} z_{\pi(i)}\right) \prod_{i \geq \pi(i)} \varphi_{\lambda_{i}}\left(q \bar{\lambda}_{i}^{-1} z_{\pi(i)}\right)\right]
$$

where $\varphi_{k}(z):=(z-1)\left(z-q^{-1}\right) \ldots\left(z-q^{-k+1}\right)$. Since $I_{S}$ is $\Xi_{i}$-stable there is $E_{\lambda^{\prime}} \in I_{S}$ with $E_{\lambda^{\prime}}(\lambda) \neq 0$. In particular, $\left|\lambda^{\prime}\right| \leq|\lambda|$. On the other hand, $E_{\lambda^{\prime}}\left(\overline{\lambda^{\prime}}\right) \neq 0$ implies $\lambda^{\prime} \in S$, i.e. $\lambda \preccurlyeq \lambda^{\prime}$. Therefore, $\lambda^{\prime}=\lambda$ (Lemma 4.1).

4.6. Theorem. Let $S \subseteq \Lambda$ be closed. Then $I_{S}=\oplus_{\lambda \in S} k E_{\lambda}$.

Proof. By Theorem 4.4, we have $I_{S}=\oplus_{\lambda \in S^{\prime}} k E_{\lambda}$ for some subset $S^{\prime} \subseteq \Lambda$. Let $\lambda \in S^{\prime}$. Then $E_{\lambda}(\bar{\lambda}) \neq 0$ implies $\lambda \in S$, hence $S^{\prime} \subseteq S$. Conversely, let $\lambda \in S$ and $\mu \in \Lambda \backslash S$. Then $E_{\lambda}(\bar{\mu})=0$ by the extra vanishing theorem. Hence $E_{\lambda} \in I_{S}$ and $\lambda \in S^{\prime}$.

4.7. Corollary. Let $E_{\lambda} E_{\mu}=\sum_{\nu} c_{\lambda \mu}^{\nu} E_{\nu}$. Then $c_{\lambda \mu}^{\nu}=0$ unless $\lambda, \mu \preccurlyeq \nu$.

Proof. Let $S$ be the set of $\nu \in \Lambda$ with $\lambda \preccurlyeq \nu$. Then by Theorem 4.5 and Theorem 4.6, the principal ideal $\mathcal{P} E_{\lambda}$ is contained in $\oplus_{\nu \in S} k E_{\nu}$. This shows $\lambda \preccurlyeq \nu$ whenever $c_{\lambda \mu}^{\nu} \neq 0$. The relation $\mu \preccurlyeq \nu$ follows by symmetry.

The whole discussion has also a symmetric counterpart. As already mentioned, on $\Lambda^{+}$the order relation $\preccurlyeq$ is just inclusion of diagrams. Then everything works for this order relation. See $[\mathrm{KS}]$ for the precise statements.

\section{The inversion formula and integrality results}

We have seen that the Macdonald polynomials are obtained from the $E_{\lambda}$ or $P_{\lambda}$ by taking the top homogeneous part. In this section we show how to invert this process.

The Capelli polynomials $E_{\lambda}$ and the Macdonald polynomials $\bar{E}_{\lambda}$ form two bases of $\mathcal{P}$. Define $\Psi \in \operatorname{End}_{k} \mathcal{P}$ by $\Psi\left(\bar{E}_{\lambda}\right)=E_{\lambda}$. Another way to describe $\Psi$ is as follows: 
Let $\mathcal{P}_{d}$ be the set of polynomials of degree $d$ which vanish in $S(n, d-1)$ and let $\overline{\mathcal{P}}_{d}$ the set of homogeneous polynomials of degree $d$. Then taking the top degree term gives an isomorphism $\Psi^{\prime}: \mathcal{P}_{d} \stackrel{\sim}{\rightarrow} \overline{\mathcal{P}}_{d}$. Since $\Psi^{\prime}$ maps $E_{\lambda}$ to $\bar{E}_{\lambda}$ we see $\Psi^{\prime}=\Psi^{-1}$.

The next theorem tells us that Capelli and Macdonald polynomials are essentially the same up to base change by $\Psi$.

5.1. Lemma. The following table gives for an operator $A$ its intertwined operator $\Psi A \Psi^{-1}$.

\begin{tabular}{lllll}
$A$ & $H_{i}$ & $\xi_{i}^{-1}$ & $\bar{\Phi}:=z_{n} \Delta$ & $z_{i}$ \\
\hline$\Psi A \Psi^{-1}$ & $H_{i}$ & $\Xi_{i}$ & $\Phi$ & $z_{i}-\Xi_{i}^{-1}$
\end{tabular}

In particular, the operators $z_{i}-\Xi_{i}^{-1}$ commute pairwise.

Proof. The endomorphism $\Psi$ maps an eigenvectors of $\xi_{i}^{-1}$ to an eigenvector of $\Xi_{i}$ with the same eigenvalue. Thus we obtain $\Psi \xi_{i}^{-1}=\Xi_{i} \Psi$. For $f \in \mathcal{P}_{d}$ holds $H_{i} f \in \mathcal{P}_{d}$ and $\Psi^{\prime}\left(H_{i} f\right)=H_{i} \Psi^{\prime}(f)$. Hence, $H_{i}$ commutes with $\Psi$.

We have $\Phi\left(\mathcal{P}_{d}\right) \subseteq \mathcal{P}_{d+1}$ (Corollary 2.5) and therefore $\Psi^{\prime}(\Phi(f))=\bar{\Phi}\left(\Psi^{\prime}(f)\right)$ for all $f \in \mathcal{P}_{d}$.

Next consider $\lambda \in \Lambda$ with $\lambda_{n} \neq 0$ and $\lambda^{*}$ as in Lemma 2.1. Then $q^{\lambda_{n}-1} \Phi\left(E_{\lambda^{*}}\right)=$ $E_{\lambda}$ and $q^{\lambda_{n}-1} \bar{\Phi}\left(\bar{E}_{\lambda^{*}}\right)=\bar{E}_{\lambda}([\mathrm{Kn}]$ Thm. 4.1). This implies $\Psi \bar{\Phi}=\Phi \Psi$.

Finally, for $z_{i}$ observe first that $\Xi_{i}$ is diagonalizable with non-zero eigenvalues. Hence $\Xi_{i}^{-1}$ exists.

According to the definition of $\Xi_{i}$, we have

$$
z_{i} \Xi_{i}-1=H_{i} \ldots H_{n-1} \Phi H_{1} \ldots H_{i-1} .
$$

Hence, by Corollary 3.3 and Theorem 3.6, the operator $z_{i}-\Xi_{i}^{-1}=\left(z_{i} \Xi_{i}-1\right) \Xi_{i}^{-1}$ maps $\mathcal{P}_{d}$ into $\mathcal{P}_{d+1}$. Looking at the top homogeneous term we see $\Psi^{\prime}\left(\left(z_{i}-\right.\right.$ $\left.\left.\Xi_{i}^{-1}\right) f\right)=z_{i} \Psi^{\prime}(f)$.

Remark. The discussion above shows that Macdonald polynomials and Capelli polynomials are just two different views of the same picture. Both have their virtues: the main structure governing Macdonald polynomials is Cherednik's scalar product on $\mathcal{P}$. By transport of structure via $\Psi$ also the Capelli polynomials are orthogonal with respect to a certain scalar product but unfortunately an explicit formula for it is not known. On the other hand, the scalar product has its replacement in the vanishing conditions which turn out to be just as good.

Observe that $\Xi_{i}^{-1}$ is no difference operator anymore. Therefore, we introduce the operator $S:=t^{-\left(\begin{array}{c}n \\ 2\end{array}\right)} \Xi_{1} \ldots \Xi_{n}$ which acts on $\mathcal{P}_{d}$ as scalar multiplication by $q^{-d}$. It intertwines with the operator $\bar{S}=\Delta^{n}$ which acts on $\overline{\mathcal{P}}_{d}$ by $q^{-d}$. Now observe that $Z_{i}:=\left(z_{i}-\Xi_{i}^{-1}\right) S$ is a difference operator which corresponds on the homogeneous side to $z_{i} \bar{S}=q \bar{S} z_{i}$. 
Now we can state the inversion formula:

5.2. Theorem. a) The operators $Z_{1}, \ldots, Z_{n}$ commute pairwise.

b) Let $f$ be homogeneous of degree d. Then $\Psi(f)=q^{\left(\begin{array}{l}2 \\ 2\end{array}\right)} f\left(Z_{1}, \ldots, Z_{n}\right) \cdot 1$ (where $1 \in \mathcal{P}$ denotes the unity).

Proof. Since $f$ is homogeneous of degree $d$, we obtain $f=q^{\left(\begin{array}{l}d \\ 2\end{array}\right)} f\left(z_{i} \bar{S}\right) \cdot 1$. Thus b) follows by applying $\Psi$.

This formula can be used to lift results for Macdonald polynomials to Capelli polynomials. As an example, we investigate integrality properties.

For this we use a different normalization of $E_{\lambda}$. Recall, that the diagram of $\lambda \in \Lambda$ is the set of points (usually called boxes) $s=(i, j) \in \mathbb{Z}^{2}$ such that $1 \leq i \leq n$ and $1 \leq j \leq \lambda_{i}$. For each box $s$ we define the arm-length $a(s)$ and leg-length $l(s)$ as

$$
\begin{aligned}
a(s) & :=\lambda_{i}-j \\
l^{\prime}(s) & :=\#\left\{k=1, \ldots, i-1 \mid j \leq \lambda_{k}+1 \leq \lambda_{i}\right\} \\
l^{\prime \prime}(s) & :=\#\left\{k=i+1, \ldots, n \mid j \leq \lambda_{k} \leq \lambda_{i}\right\} \\
l(s) & :=l^{\prime}(s)+l^{\prime \prime}(s)
\end{aligned}
$$

If $\lambda \in \Lambda^{+}$is a partition then $l^{\prime}(s)=0$ and $l^{\prime \prime}(s)=l(s)$ is just the usual leg-length. We define

$$
\begin{aligned}
\mathcal{E}_{\lambda} & :=\prod_{s \in \lambda}\left(1-q^{a(s)+1} t^{l(s)+1}\right) E_{\lambda} . \\
\mathcal{P}_{\lambda} & :=\prod_{s \in \lambda}\left(1-q^{a(s)} t^{l(s)+1}\right) P_{\lambda} .
\end{aligned}
$$

With this normalization, we obtain:

5.3. Proposition. The coefficients of $\mathcal{E}_{\lambda}$ and $\mathcal{P}_{\lambda}$ are in $\mathbb{Z}\left[q, q^{-1}, t, t^{-1}\right]$.

Proof. The leading terms $\overline{\mathcal{E}}_{\lambda}$ and $\overline{\mathcal{P}}_{\lambda}$ have coefficients in $\mathbb{Z}[q, t]$ by Corollary 5.2 and Theorem 6.1 of $[\mathrm{Kn}]$. The result follows from the fact that the operators $Z_{i}$ are defined over $\mathbb{Z}\left[q, q^{-1}, t, t^{-1}\right]$.

This result can be improved. For $m=1, \ldots, n$ we define the operators

$$
\begin{aligned}
& A_{m}:=H_{m} H_{m+1} \ldots H_{n-1} \Phi \\
& \bar{A}_{m}:=\bar{H}_{m} \bar{H}_{m+1} \ldots \bar{H}_{n-1} \Phi
\end{aligned}
$$

Then we have the following recursion relation:

5.4. Theorem. Let $\lambda \in \Lambda$ with $m:=l(\lambda)>0$. Put $\lambda^{*}:=\left(\lambda_{m}-1, \lambda_{1}, \ldots\right.$, $\left.\lambda_{m-1}, 0, \ldots, 0\right)$. Then $\mathcal{E}_{\lambda}=q^{\lambda_{m}-1}\left[\bar{A}_{m}-\bar{\lambda}_{m} t^{m} A_{m}\right] \mathcal{E}_{\lambda^{*}}$. 
Proof. Apply $\Psi$ to both sides of the corresponding formula in [Kn] Theorem 5.1.

5.5. Corollary. Let $\mathcal{E}_{\lambda}=\sum_{\mu} e_{\lambda \mu} z^{\mu}$ and $\mathcal{P}_{\lambda}=\sum_{\mu} p_{\lambda \mu} m_{\mu}$. Then

$$
e_{\lambda \mu}, p_{\lambda \mu} \in t^{-(n-1)(|\lambda|-|\mu|)} \mathbb{Z}[q, t]
$$

Proof. The operators $H_{i}$ and $\bar{H}_{i}$ are defined over $\mathbb{Z}[t]$. Since $\Phi=\left(z_{n}-t^{-n+1}\right) \Delta$, the recursion formula implies $e_{\lambda \mu} \in t^{-k} \mathbb{Z}\left[q, q^{-1}, t\right]$ with $k=(n-1)(|\lambda|-|\mu|)$. To show that no negative powers of $q$ appear observe that $z_{1} \Xi_{1}-1=H_{1} \ldots H_{n-1} \Phi$. Therefore,

$$
q^{\lambda_{m}-1} \Phi\left(E_{\lambda^{*}}\right)=q^{\lambda_{m}-1} H_{n-1}^{-1} \ldots H_{1}^{-1}\left(z_{1}\left(\bar{\lambda}_{1}^{*}\right)^{-1}-1\right) \mathcal{E}_{\lambda^{*}} .
$$

The claim follows from $\bar{\lambda}_{1}^{*}=q^{\lambda_{m}-1} t^{-k}$ for some $k \in \mathbb{N}$.

The assertion for $\mathcal{P}_{\lambda}$ follows by symmetrization as in the proof of [Kn] Theorem 6.1 .

Remark. The assertion of the last theorem is equivalent to

$$
t^{(n-1)|\lambda|} \mathcal{E}_{\lambda}\left(t^{1-n} z_{1}, \ldots, t^{1-n} z_{n}\right) \in \mathbb{Z}\left[q, t, z_{i}\right]
$$

\section{The classical limit}

Let $\alpha$ be a formal parameter. If one puts $q=t^{\alpha}$ and lets $t$ go to 1 then $(1-t)^{-|\lambda|} \overline{\mathcal{P}}_{\lambda}$ converges to the Jack polynomial $J_{\lambda}^{(\alpha)}(z)$. In this final section, I will discuss the analogue for our non-homogeneous Macdonald polynomials. For this it is a bit more convenient to set $t=q^{r}$ and let $q$ tend to 1 . Then $\alpha$ and $r$ are related by $\alpha=1 / r$.

We introduce the following notation: Let $p(q, t) \in \mathbb{Q}(q, t), p_{0} \in \mathbb{Q}$ and $k \in \mathbb{N}$. Then we write $p \stackrel{k, r}{\longrightarrow} p_{0}$ if $\lim _{q \rightarrow 1} \frac{p\left(q, q^{r}\right)}{(q-1)^{k}}=p_{0}$. For example, $q^{a} t^{b}-1 \stackrel{1, r}{\longrightarrow} a+b r$. (In purely algebraic terms, $\lim _{q \rightarrow 1} p\left(q, q^{r}\right)$ means: write $p(q, t)$ in terms of $x:=q-1$ and $r:=\frac{t-1}{q-1}$ and put $x=0$.)

As $q \rightarrow 1$, the points $\bar{\lambda}$ all collapse to 1 . Therefore, we introduce the function $\varphi_{q}(x):=\frac{x-1}{q-1}$ and the affine transformation $\varphi_{q}(z):=\left(\varphi_{q}\left(z_{1}\right), \ldots, \varphi_{q}\left(z_{n}\right)\right)$. Set $\tilde{\varrho}:=(0,-r, \ldots-(n-1) r)$. Then we have $\varphi_{q}(\bar{\lambda}) \stackrel{0, r}{\longrightarrow} \tilde{\lambda}$ where $\tilde{\lambda}:=\lambda+w_{\lambda} \varrho$. Conversely, we can write $\bar{\lambda}=q^{\tilde{\lambda}}$. 
The action of $\varphi_{q}$ on $\mathcal{P}$ is given by $\varphi_{q} f(z)=f\left(\varphi_{q}^{-1}(z)\right)$. We extend our notation as follows: if $X(q, t)$ and $X(r)$ are operators on $\mathcal{P}$ then we write $X(q, t) \stackrel{k, r}{\longrightarrow} X(r)$ if for all $f \in \mathcal{P}$

$$
\lim _{q \rightarrow 1}(q-1)^{-k} \varphi_{q} X\left(q, q^{r}\right) \varphi_{q}^{-1} f=X(r) f
$$

For example, if $X(q, t)$ is the multiplication operator by $g(z ; q, t)$ then $\varphi_{q} X\left(q, q^{r}\right) \varphi_{q}^{-1}$ is multiplication by $\varphi_{q} g=g \circ \varphi_{q}^{-1}$. In this way, $g \stackrel{k, r}{\longrightarrow} g_{0}$ has to be understood. Since $\varphi_{q}^{-1}(x)=(q-1) x+1$ we obtain, for example, $z_{i} \stackrel{0, r}{\longrightarrow} 1$ while $z_{i}-1 \stackrel{1, r}{\longrightarrow} z_{i}$.

Define the operators $\tilde{\Delta} f(z):=f\left(z_{n}-1, z_{1}, \ldots, z_{n-1}\right)$ and $\tilde{\Phi}:=\left(z_{n}+(n-1) r\right) \tilde{\Delta}$.

6.1. Lemma. We have $\Delta \stackrel{0, r}{\longrightarrow} \tilde{\Delta}$ and $\Phi \stackrel{1, r}{\longrightarrow} \tilde{\Phi}$.

Proof. We have $\varphi_{q} \Delta \varphi_{q}^{-1} f(z)=f\left(\psi\left(z_{n}\right), z_{1}, \ldots, z_{n-1}\right)$ where $\psi(z)=z_{n} / q-$ $1 / q \stackrel{0, r}{\longrightarrow} z_{n}-1$. Moreover, $z_{n}\left(\varphi_{q}^{-1}(z)\right)-t^{-n+1}=(q-1) z_{n}+\left(1-t^{-n+1}\right) \stackrel{1, r}{\longrightarrow} z_{n}+$ $(n-1) r$.

6.2. Theorem. For every $\lambda \in \Lambda$ there is a unique polynomial $\tilde{E}_{\lambda}=$ of degree $|\lambda|$ and $z^{\lambda}$-coefficient equal to 1 which vanishes at all $\tilde{\mu}$ with $|\mu| \leq|\lambda|$ and $\mu \neq \lambda$. Moreover, $E_{\lambda} \stackrel{|\lambda|, r}{\longrightarrow} \tilde{E}_{\lambda}$.

Proof. Repeat the proof of Theorem 2.2 with respect to vanishing at $\varphi_{q}(\bar{\lambda})$. Then one sees, by induction, that the limit $q \rightarrow 1$ exists.

In the limit $q \rightarrow 1$, the symmetric version has been already treated in [KS]. As above we obtain

6.3. Theorem. For every $\lambda \in \Lambda^{+}$there is a unique polynomial $\tilde{P}_{\lambda}$ of degree $|\lambda|$ which vanishes at all $\tilde{\mu}=\mu+\varrho$ where $\mu \in \Lambda^{+}$with $|\mu| \leq|\lambda|$ and $\mu \neq \lambda$. Moreover, $P_{\lambda} \stackrel{|\lambda|, r}{\longrightarrow} \tilde{P}_{\lambda}$.

Next we study the limit of the Hecke operators. Let $\sigma_{i}:=s_{i}-r N_{i}$.

6.4. Lemma. We have $H_{i}, \bar{H}_{i} \stackrel{0, r}{\longrightarrow} \sigma_{i}$ and $H_{i}-\bar{H}_{i} \stackrel{1, r}{\longrightarrow} r$. 
Proof. First observe that $s_{i}$ commutes with $\varphi_{q}$. Moreover,

$$
(1-t) \frac{z_{i+1}}{z_{i}-z_{i+1}} \varphi_{q}^{-1}=\frac{1-t}{q-1} \frac{(q-1) z_{i+1}+1}{z_{i}-z_{i+1}} \stackrel{0, r}{\longrightarrow} \frac{-r}{z_{i}-z_{i+1}} .
$$

This implies the claim for $\bar{H}_{i}$. For $H_{i}$, use $H_{i}=\bar{H}_{i}+t-1$.

The braid relations for the $H_{i}$ imply them for the $\sigma_{i}$. Moreover, from $H_{i} \bar{H}_{i}=t$ we deduce $\sigma_{i}^{2}=1$. Hence we obtain

6.5. Corollary. The mapping $s_{i} \mapsto \sigma_{i}$ extends uniquely to an action of $W$ on $\mathcal{P}$.

Remark. It is not difficult to see that the standard action of $W$ and the one defined above are conjugated by an element $U \in \operatorname{End}_{\mathcal{P} W} \mathcal{P} \cong M_{n !}\left(\mathcal{P}^{W}\right)$. It would be interesting to find such a $U$ explicitly. Note however that $U$ is not uniquely determined.

Observe that the commutation relations $z_{i+1} H_{i}=\bar{H}_{i} z_{i}$ can we rewritten as $\left(z_{i+1}-1\right) H_{i}=\bar{H}_{i}\left(z_{i}-1\right)-\left(H_{i}-\bar{H}_{i}\right)$ which implies

$$
z_{i+1} \sigma_{i}=\sigma_{i} z_{i}-r ; \quad z_{j} \sigma_{i}=\sigma_{i} z_{j}, \quad j \neq i, i+1 .
$$

Therefore, the $\sigma_{i}$ and $z_{j}$ generate a graded Hecke algebra.

We now consider the limit of the Cherednik operators. Let

$$
\tilde{\Xi}_{i}:=z_{i}-\sigma_{i} \ldots \sigma_{n-1} \tilde{\Phi} \sigma_{1} \ldots \sigma_{i-1} \text {. }
$$

Then we have

6.6. Theorem. For $i=1, \ldots, n$ holds $\Xi_{i}-1 \stackrel{1, r}{\longrightarrow}-\tilde{\Xi}_{i}$. Moreover, $\tilde{\Xi}_{i} \tilde{E}_{\lambda}=\tilde{\lambda}_{i} \tilde{E}_{\lambda}$ for all $\lambda \in \Lambda$. In particular, the $\tilde{\Xi}_{i}$ commute pairwise.

Proof. Follows from $z_{i}^{-1}-1 \stackrel{1, r}{\longrightarrow}-z_{i}, H_{i} \stackrel{0, r}{\longrightarrow} \sigma_{i}, \Phi \stackrel{1, r}{\longrightarrow} \tilde{\Phi}$, and $\bar{\lambda}^{-1}-1 \stackrel{1, r}{\longrightarrow}-\tilde{\lambda}$.

6.7. Corollary. The top homogeneous part of $\tilde{P}_{\lambda}$ and $\tilde{E}_{\lambda}$ is a Jack polynomial and Opdam's non-symmetric analogue $[\mathrm{Op}]$, respectively.

The extra vanishing theorem goes through verbatim:

6.8. Theorem. Let $\lambda, \mu \in \Lambda$ with $\lambda \npreceq \mu$. Then $\tilde{E}_{\lambda}(\tilde{\mu})=0$. 
For the inversion formula we introduce

$$
\tilde{Z}_{i}:=z_{i}-\tilde{\Xi}_{i}=\sigma_{i} \ldots \sigma_{n-1} \tilde{\Phi} \sigma_{1} \ldots \sigma_{i-1} .
$$

Furthermore, let $\tilde{\Psi}: \mathcal{P} \rightarrow \mathcal{P}$ be the linear automorphism which maps the leading term of $\tilde{E}_{\lambda}$ to $\tilde{E}_{\lambda}$. Then we obtain

6.9. Theorem. We have $Z_{i} \stackrel{1, r}{\longrightarrow} \tilde{Z}_{i}$. Moreover, $\tilde{Z}_{i} \tilde{\Psi}=\tilde{\Psi} z_{i}$ and the inversion formula holds: $\Psi(f)=f\left(\tilde{Z}_{1}, \ldots, \tilde{Z}_{n}\right) \cdot 1$ for all $f \in \mathcal{P}$.

Finally, as for integrality, we choose the following normalizing factors:

$$
\begin{gathered}
\tilde{\mathcal{E}}_{\lambda}:=\prod_{s \in \lambda}((a(s)+1)+(l(s)+1) r) \tilde{E}_{\lambda} . \\
\tilde{\mathcal{P}}_{\lambda}:=\prod_{s \in \lambda}(a(s)+(l(s)+1) r) \tilde{P}_{\lambda}
\end{gathered}
$$

With this normalization, we obtain:

6.10. Theorem. We have $\mathcal{E}_{\lambda} \stackrel{2|\lambda|, r}{\longrightarrow}(-1)^{|\lambda|} \tilde{\mathcal{E}}_{\lambda}$ and $\mathcal{P}_{\lambda} \stackrel{2|\lambda|, r}{\longrightarrow}(-1)^{|\lambda|} \tilde{\mathcal{P}}_{\lambda}$. Moreover, the coefficients of $\mathcal{E}_{\lambda}$ and $\mathcal{P}_{\lambda}$ are in $\mathbb{Z}[r]$.

\section{References}

[Ch] I. Cherednik, Non-symmetric Macdonald's polynomials, Preprint, 1995, 25 pages.

[HU] R. Howe and T. Umeda, The Capelli identity, the double commutant theorem, and multiplicity-free actions, Math. Ann. 290 (1991), 569-619.

[Kn] F. Knop, Integrality of two variable Kostka functions, Preprint, 1996, 12 pages.

[KS] F. Knop and S. Sahi, Difference operators and symmetric functions defined by their zeros, Preprint, 1996, 14 pages.

[M1] I. Macdonald, Symmetric functions and Hall polynomials, 2nd ed., Clarendon Press, Oxford 1995.

[M2] I. Macdonald, Affine Hecke algebras and orthogonal polynomials, Séminaire Bourbaki $\mathbf{7 9 7}$ (1995).

[Ok] A. Okounkov, Quantum immanants and higher Capelli identities, Preprint, 1995, 23 pages.

[Ol] G. Olshanski, Quasi-symmetric functions and factorial Schur functions, Preprint, 1995, 21 pages.

[Op] E. Opdam, Harmonic analysis for certain representations of graded Hecke algebras, Acta Math. 175 (1995), 75-121.

[Sa] S. Sahi, The spectrum of certain invariant differential operators associated to Hermitian symmetric spaces, In: Lie theory and geometry, J.-L. Brylinski et al. (Eds.), Progress Math. 123 Birkhäuser, Boston 1994, 569-576.

[WUN] M. Wakayama, T. Umeda and M. Noumi, A quantum analogue of the Capelli identity and an elementary differential calculus on $G L_{q}(n)$, Duke Math. J. 76 (1994), 567-594. 
Friedrich Knop

Department of Mathematics

Rutgers University

New Brunswick NJ

08903

USA

knop@math.rutgers.edu

(Received: April 1, 1996 ) 